\title{
CT Differentiation of Cholangiocarcinoma from Periductal Fibrosis in Patients with Hepatolithiasis
}

\author{
Hee Sun Park ${ }^{1}$ \\ Jeong Min Lee \\ Se Hyung Kim \\ Jun Yong Jeong \\ Young Jun Kim \\ Kyoung Ho Lee \\ Seung Hong Choi \\ Joon Koo Han \\ Byung Ihn Choi
}

Keywords: biliary system, cholangiocarcinoma, CT, fibrosis, hepatolithiasis, liver

\section{DOI:10.2214/AJR.05.0247}

Received February 12, 2005; accepted after revision June 7, 2005.

${ }^{1}$ All authors: Department of Radiology and Institute of Radiation Medicine, Seoul National University College of Medicine, 28, Yongon-dong, Chongno-gu, Seoul 110-744, Korea. Address correspondence to J. M. Lee

(leejm@radcom.snu.ac.kr).

AJR 2006; 187:445-453

0361-803X/06/1872-445

(C) American Roentgen Ray Society

OBJECTIVE. The objective of our study was to determine useful CT findings for differentiating cholangiocarcinoma from periductal fibrosis in patients with hepatolithiasis.

MATERIALS AND METHODS. CT images of 30 patients with hepatolithiasis and pathologically proven cholangiocarcinoma $(n=14)$ or periductal fibrosis $(n=16)$ were retrospectively reviewed. Helical CT scans were obtained before, 30 seconds after, and 65 seconds after the start of contrast material injection. Analysis of CT findings included evaluation for the presence of periductal soft-tissue density, bile duct wall thickening at the stricture site, ascites, portal vein obliteration, lymph node enlargement, and a duct stone; assessment of the degree of ductal dilatation; and evaluation of the enhancement pattern of periductal lesions, thickened ductal wall, and hepatic parenchyma. The CT attenuation coefficients of the thickened ductal wall and adjacent normal-looking bile duct were measured on images obtained during each phase. Among these findings, statistically significant variables were determined using the Fisher's exact test and Student's $t$ test. Sensitivity and specificity values of the CT criteria were also calculated.

RESULTS. The presence of periductal soft-tissue density $(p=0.002)$, higher enhancement of the duct than adjacent bile duct on portal venous phase images $(p=0.008)$, ductal wall thickening $(p=0.026)$, portal vein obliteration $(p=0.031)$, and lymph node enlargement $(p=0.031)$ were found to be the significant findings for differentiating cholangiocarcinoma from fibrosis in patients with hepatolithiasis. When any two or more of these five criteria were used in combination, we could identify $100 \%$ of the patients with cholangiocarcinoma but only $12.5 \%$ of the patients with fibrosis.

CONCLUSION. Cholangiocarcinoma in patients with hepatolithiasis can be diagnosed using specific CT criteria.

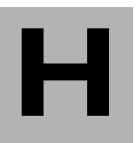

epatolithiasis is a disease characterized by intrahepatic pigmented stones and recurrent attacks of abdominal pain, fever, chills, and jaundice $[1,2]$. It is also known by various synonyms such as oriental cholangiohepatitis, intrahepatic pigmented calculus disease, and recurrent pyogenic cholangitis. The cause of hepatolithiasis is unclear, but associations with clonorchiasis, ascariasis, bacterial infection, biliary stasis, and nutritional deficiency have been noted [1-3]. Histologically, there are inflammatory and fibrotic changes in the bile duct walls, periportal spaces, and hepatic parenchyma that lead to stricture formation $[3,4]$. Men and women are affected almost equally, and the greatest number of cases occur in persons who are 20-40 years old.

The natural history of the disease is marked by recurrent attacks of cholangitis, usually once or twice a year $[2,3]$. Because of the re- calcitrant nature of the strictures and stones, the complications of the condition-that is, cholangitis, abscess, and jaundice-are relentless. Repeated surgery for recurrent cholangitis and abscess formation due to re-formed or residual stones results in substantial morbidity and mortality $[3,5]$. The incidence of hepatolithiasis is known to be high throughout East Asia; hepatolithiasis is rare in Europe and the United States, but the recent pattern of increased population migration to the West has led to the increased prevalence and recognition of hepatolithiasis in Western countries [5].

According to previous studies, hepatolithiasis is known to be associated with cholangiocarcinoma, and the incidence rates of cholangiocarcinoma in patients with hepatolithiasis have been reported in the range of 5-6\% [6-9]. Alternatively, the percentage of patients with cholangiocarcinoma with concomitant hepatolithiasis has been reported to be in the range 


\section{Park et al.}

of $17-27 \%$ [10-12]. Chronic irritation by intrahepatic calculi, bile stasis, and bacterial infection have been proposed as possible factors responsible for its development [6]. A careful search for the presence of cholangiocarcinoma is crucial in the treatment of patients with hepatolithiasis. However, because the clinical features of hepatolithiasis have been reported to be similar in patients with or without the coexistence of cholangiocarcinoma, preoperative imaging workup, especially with CT, is important for the selection of appropriate surgical therapy and treatment planning [6].

Typical CT findings of cholangiocarcinoma are parenchymal low-attenuated mass with rim enhancement, capsular retraction and delayed enhancement, biliary duct wall thickening, or intraductal polypoid mass with dilatation of upstream ducts [13-16]. However, only a few reports describe the radiologic findings of cholangiocarcinoma occurring in patients with hepatolithiasis [17-19]. In patients with hepatolithiasis, fibrotic masses in the bile duct walls and periductal hepatic parenchyma are often seen as a form of periductal tumorlike lesions and may appear similar to cholangiocarcinoma on CT [19]. Furthermore, in patients with hepatolithiasis, the stricture or stenosis caused by cholangiocarcinoma may not be easy to differentiate from that caused by stones $[6,10,20]$.

Despite the fact that differentiation between periductal fibrosis and cholangiocarcinoma in patients with hepatolithiasis is important because of their different prognoses, no report, to our knowledge, has compared the CT findings of cholangiocarcinoma occurring in the background of hepatolithiasis with those of fibrosis. The purpose of this study was to identify the useful CT findings for differentiating cholangiocarcinoma manifesting as periductal lesions from periductal fibrosis in patients with hepatolithiasis.

\section{Materials and Methods Patient Selection}

From a computerized search of our hospital's database of medical records between January 1997 and March 2003, we found a total of 246 patients diagnosed with hepatolithiasis. All of these patients clinically presented with recurrent attacks of fever, chills, abdominal pain, and jaundice and radiologic evidence of intrahepatic stones or other findings suggesting hepatolithiasis, such as biliary obstruction or atrophy of a hepatic segment. Among these patients, we selected patients for our study on the basis of the following inclusion criteria: available multiphase helical CT examination; suspected focal intrahepatic duct stricture on CT; and histologic confirmation of involved ductal pathology, either surgically or by biopsy.

Finally, 30 patients were included in our retrospective study - that is, 14 patients with cholangiocarcinoma (male-to-female ratio, 7:7; mean age, 59.6 years; age range, $45-74$ years) and 16 patients with periductal fibrosis (male-to-female ratio, 9:7; mean age, 52.9 years; age range, 41-71 years). Cholangiocarcinoma was proven by percutaneous sonographically guided liver biopsy $(n=4)$, choledochoscopic biopsy $(n=5)$, or surgery $(n=3$ [left lobectomy in two patients and left lateral segmentectomy in one]). Peritoneoscopic and open omental biopsy were performed on each of the remaining two patients, and they were proven to have metastatic adenocarcinoma clinically from the liver. All 16 patients with fibrosis underwent hepatic surgery: left lateral segmentectomy $(n=8)$, left lobectomy $(n=7)$, or right lobectomy $(n=1)$. For the limited and anonymous review of these patients' data for this study, we were not required to have formal approval or informed patient consent according to the institutional review board of our hospital.

\section{CT Examination}

The CT examinations evaluated for this study were performed on different helical CT scanners (Somatom Plus 4, Siemens Medical Solutions; or HighSpeed, GE Healthcare) using the following parameters: 5-mm collimation, 5-mm reconstruction interval, and a 1:1 table pitch. Transverse images were reconstructed with a soft-tissue algorithm. The X-ray tube voltage used was 120-140 $\mathrm{kV}$, and the current varied between 240 and 260 $\mathrm{mA}$. The CT images were routinely obtained with the patient in a supine position during full inspiration. Both unenhanced and contrast-enhanced CT scans were obtained. With IV injection of $120 \mathrm{~mL}$ of nonionic contrast material (iopromide [Ultravist 370 , Schering]), both hepatic artery phase (HAP) and portal venous phase (PVP) images were obtained with a scanning delay of 30 and 65 seconds, respectively. Contrast material was administered at a rate of $3 \mathrm{~mL} / \mathrm{s}$ using a mechanical power injector (CT 9000 ADV Digital Injection System, LiebelFlarsheim) through an 18-gauge angiographic catheter inserted into a forearm vein.

\section{CT Analysis}

CT scan data were available on a PACS, and all images were reviewed at a PACS monitor. CT scans were reviewed retrospectively by two experienced abdominal radiologists (15 and 7 years of experience) in consensus who were blinded to the final pathology results. For the analysis of the CT features, cases of cholangiocarcinomas and benign fibrotic masses were randomly intermixed. For subjective analysis, the following CT findings were included: periductal soft-tissue-density lesion and its enhancement pattern; ductal wall thickening at the stricture site and, if present, its extent and its enhancement pattern; degree of ductal dilatation proximal to the stricture site; presence of intrahepatic duct or common bile duct stone; portal vein patency; ascites; and lymph node enlargement. A periductal soft-tissue-density lesion was defined as a low-attenuated lesion compared with adjacent liver parenchyma along the involved bile duct on contrast-enhanced CT. Relative enhancement was obtained by calculating the absolute value of attenuation of the periductal soft-tissue density subtracted by that of adjacent hepatic parenchyma. Ductal dilatation proximal to the stricture was defined as mild ( $\leq 15 \mathrm{~mm}$ in diameter) or marked (> $15 \mathrm{~mm}$ in diameter) [21]. Portal vein status was analyzed as one of the following three categories: intact; narrowed, but patent; or obliterated. A lymph node was considered to be significantly enlarged when the short diameter exceeded $1 \mathrm{~cm}$ or it had a nonenhancing, low-attenuated portion on PVP images, thereby suggesting necrosis even if the diameter was less than $1 \mathrm{~cm}$.

To assess the degree of enhancement of the ductal wall at the stricture site, the attenuation of the ductal lesion and the normal bile duct wall were measured (in Hounsfield units $[\mathrm{H}]$ ) by one of the authors using circular regions of interest (ROIs) on unenhanced, HAP, and PVP images. The ROI cursors were carefully placed to encompass as much of the ductal wall as possible and to avoid adjacent structures; the mean size of the ROI cursors was 10 $\mathrm{mm}^{2}$ (range, $6-15 \mathrm{~mm}^{2}$ ). At least three ROIs were placed on the ductal wall of the stricture site and on normal bile duct, and the ROI values were averaged as a mean ductal wall attenuation. Relative enhancement of the ductal wall was obtained by calculating the ROI value of the attenuation of the lesion subtracted from that of adjacent normal bile ductal wall. Two of the authors who were not involved in the review process measured the thickness of the ductal wall at the stricture portion on a maximally magnified CT image on the PACS monitor at our institute with electronic calipers.

\section{Statistical Analysis}

The Kolmogorov-Smirnov test was used to determine whether continuous variables followed a normal distribution. Sensitivity and specificity analyses were performed for a range of ductal wall thicknesses and relative ductal wall enhancement values to generate a receiver operating characteristic (ROC) curve [22] and to determine the optimal cutoff of ductal wall thickness and relative ductal enhancement for the detection of cholangiocarcinoma in patients with hepatolithiasis. The optimal cutoff point was defined as the value at which the 


\section{CT Diagnosis of Cholangiocarcinoma Versus Periductal Fibrosis}

sum of the sensitivity and the specificity was maximized. From the subjective analysis regarding the imaging findings of the cholangiocarcinoma and fibrosis groups, statistically significant variables were determined using the Fisher's exact test and Student's $t$ test. A $p$ value of less than 0.05 was required for rejection of the null hypothesis. Also, the sensitivity and specificity values of each of the CT criteria were calculated. Numbers used in this statistical analysis were the numbers of lesions. For statistical analysis, we used SPSS for Microsoft Windows (version 10.0, SPSS) and MedCalc for Windows (version 8.0.0.1, MedCalc).

\section{Results}

Table 1 summarizes the enhancement characteristics of the thickened ductal wall and the thickness of the ductal wall. Continuous variables followed a normal distribution $(p>0.05$, Kolmogorov-Smirnov test). The enhancement value and relative enhancement value in the PVP only were significantly different in the two groups ( $p=0.011$ and 0.006 , respectively; Student's $t$ test). The difference in ductal wall thickness was also significant $(p=0.008$, Student's $t$ test). The ROC curves and scatterplots to determine the optimal cutoff value of the relative ductal enhancement in the PVP and the ductal wall thickness for differentiating cholangiocarcinoma from periductal fibrosis are shown in Figures 1 and 2. The optimal cutoff values were $10 \mathrm{H}$ and $2.8 \mathrm{~mm}$, respectively.

Table 2 shows the different imaging features observed in cholangiocarcinoma and fibrosis in patients with hepatolithiasis. On CT scans, periductal soft-tissue-density lesions were identified in 10 patients with cholangiocarcinoma $(71.4 \%)$ and in two patients with periductal fibrosis $(12.5 \%)$ ( $p=0.002$, Fisher's exact test) (Fig. 3). As for the enhancement pattern of periductal soft-tissue-density lesions

TABLE I: Enhancement Characteristics and Wall Thickness of Ductal Lesions

\begin{tabular}{l|c|c|c}
\hline \multirow{2}{*}{ Characteristic Measured } & \multicolumn{2}{|c}{ Mean Value \pm SD } \\
\cline { 2 - 3 } & Cholangiocarcinoma & \multicolumn{1}{c}{ Fibrosis } & \multicolumn{1}{c}{$p^{\mathrm{a}}$} \\
\hline Ductal wall enhancement (H) & & $36.4 \pm 14.3$ & 0.640 \\
Unenhanced & $39.1 \pm 14.1$ & $67.4 \pm 22$ & 0.054 \\
HAP & $81 \pm 14.3$ & $90.6 \pm 30.8$ & 0.011 \\
PVP & $120 \pm 27.1$ & & \\
Relative enhancement in (H) & & $3.6 \pm 24$ & 0.051 \\
HAP & $22 \pm 26$ & $4.4 \pm 25.4$ & 0.006 \\
PVP & $36.1 \pm 33.4$ & $2.3 \pm 0.9$ & 0.001 \\
Ductal wall thickness (mm) & $3.1 \pm 0.6$ & & \\
\hline
\end{tabular}

Note-HAP = hepatic arterial phase, PVP = portal venous phase.

a Student's $t$ test. of cholangiocarcinoma, all 10 cases showed low attenuation in both the HAP and the PVP and they showed progressive enhancement from the HAP to the PVP. The average value of relative enhancement on the HAP and PVP images was $24.6 \pm 14.9 \mathrm{H}$ and $45.9 \pm 19.2 \mathrm{H}$, respectively, which indicates that the lesions are seen more conspicuously on the PVP images than on the HAP images. Two cases of periductal soft-tissue-density lesions in patients with periductal fibrosis also showed low attenuation in both the HAP and PVP and progressive enhancement from the HAP to the PVP. The mean relative enhancement was $13.5 \mathrm{H}$ in the $\mathrm{HAP}$ and $44.5 \mathrm{H}$ in the PVP.

When we used $10 \mathrm{H}$ as a cutoff value indicating high ductal enhancement and 2.8-mm thickness indicating ductal wall thickening, these parameters were significant in differentiating cholangiocarcinoma from benign fibrosis (Figs. 4-6). Portal vein obliteration $(p=0.031)$ (Fig. 3) and lymph node enlargement $(p=0.031)$ (Fig. 4) were also significant parameters, and there was no necrotic lymph node in our study population. On the contrary, the degree of ductal dilatation, the presence of intrahepatic or common duct stones, and the presence of ascites were statistically insignificant in differentiating cholangiocarcinoma from benign fibrosis $(p>0.05)$.

Table 3 summarizes the sensitivity and specificity values for the diagnosis of cholangiocarcinoma in patients with hepatolithiasis. Our study results suggest that five CT findings are statistically significant in the diagnosis of cholangiocarcinoma in patients with hepatolithiasis: periductal soft-tissue-density lesion, ductal wall thickening, portal vein obliteration, lymph node enlargement, and high ductal wall enhancement in the PVP. When at least any two of these five criteria

were used in combination, we could identify all patients $(100 \%)$ with cholangiocarcinoma but only two (12.5\%) of the 16 patients with fibrosis. When four or five of these criteria were present, we achieved a specificity of $100 \%$ and a sensitivity of $21.4 \%$ (Table 4 ).

\section{Discussion}

There have been few studies about the influence of hepatolithiasis on the occurrence of cholangiocarcinoma [23]. Factors responsible for the development of cholangiocarcinoma may be partly mechanical stimuli from intrahepatic calculi and partly chemical irritation to the bile duct wall by infected bile $[4,24]$. Patients with cholangiocarcinoma generally have a poor prognosis, with an average 5-year survival rate of 5-10\% [12, 25]. Because surgery remains the only intervention offering the possibility of a cure [25], early detection of cholangiocarcinoma is essential in hepatolithiasis patients; early detection raises the chance that a patient can be included as a surgical candidate and may consequently improve their prognosis. However, the diagnosis may be easily delayed because the symptoms related to cholangiocarcinoma are vague and are also similar to those of hepatolithiasis [10, 23].

In our hospital, which is an academic center caring for a large number of patients with cancer and receives many tertiary referrals, multiphasic helical CT, including unenhanced and contrast-enhanced HAP and PVP images, is used as a main imaging study for the evaluation of patients with suspected hepatolithiasis and intrahepatic stones. Based on our experience, the challenge for radiologists interpreting these $\mathrm{CT}$ images is to differentiate cholangiocarcinoma from benign fibrotic lesions. Indeed, there have been a limited number of reports regarding the radiologic findings of cholangiocarcinoma complicating hepatolithiasis [7, 18], but there are as yet no reports published in the English-language literature. To our knowledge, no reports describing the differential points of cholangiocarcinoma that distinguish it from fibrosis in patients with hepatolithiasis have been published.

In our study, which determined the CT findings that are useful for differentiating benign stricture caused by stones from concomitant malignant strictures in patients with hepatolithiasis, several CT findings suggesting concomitant cholangiocarcinoma in patients with hepatolithiasis were found by univariate analysis including periductal soft- 


\section{Park et al.}

tissue density, ductal wall thickening, portal vein obliteration, lymph node enlargement, and a high degree enhancement of ductal wall in the PVP $(p<0.05)$. When at least two of these five signs were present, correct diagno- sis of cholangiocarcinoma could be made with a specificity of $87.5 \%$, and when at least four signs were present, diagnosis of cholangiocarcinoma could be made with a specificity of $100 \%$ (Table 4 ). We believe that the re- sults of our study are encouraging, although further research with a larger series of patients is required to test whether the findings we have noted can be successfully applied to similar patient populations.
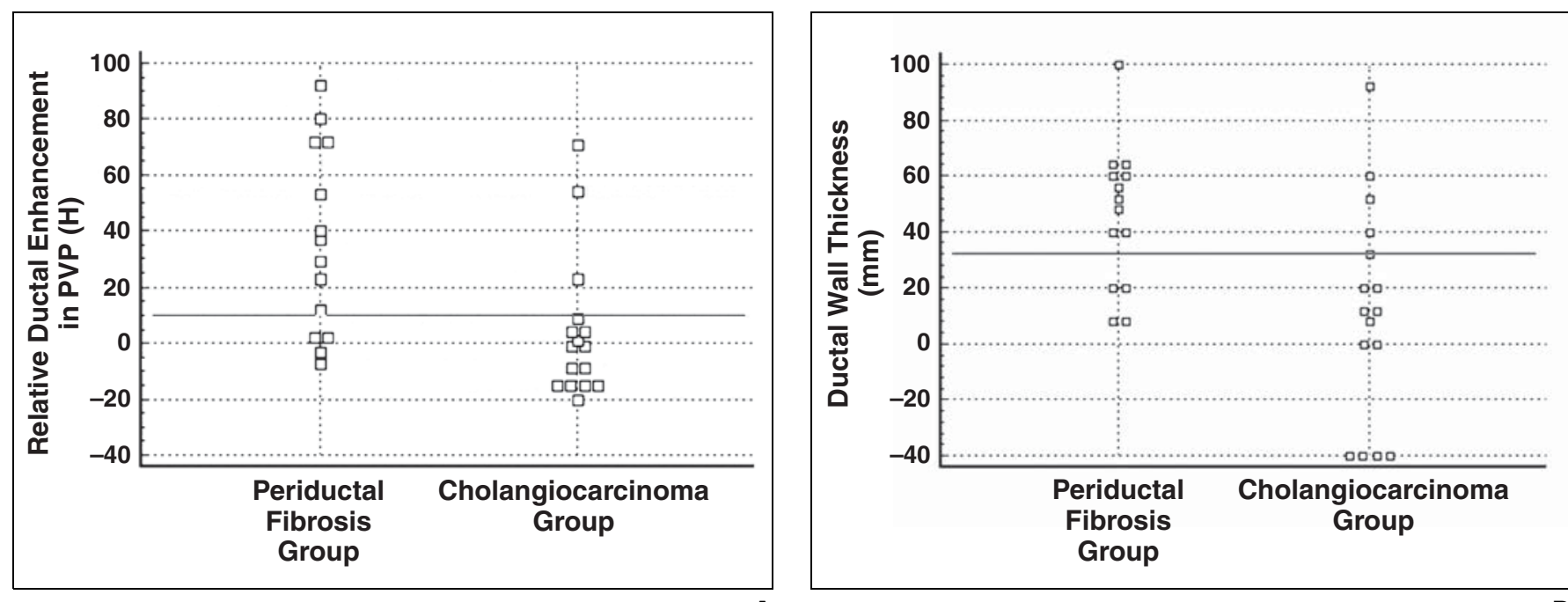

Fig. 1-Scatterplots of relative ductal enhancement.

A and $\mathbf{B}$, Scatterplots show relative ductal enhancement in portal venous phase (PVP) (A) and ductal wall thickness (B) in cholangiocarcinoma and periductal fibrosis groups. Optimal cutoff values were $10 \mathrm{H}$ and $2.8 \mathrm{~mm}$, respectively. Sensitivity and specificity were $71.4 \%$ and $81.2 \%$, respectively, at $10 \mathrm{H}$ and $71.4 \%$ and $75.0 \%$ at $2.8 \mathrm{~mm}$.
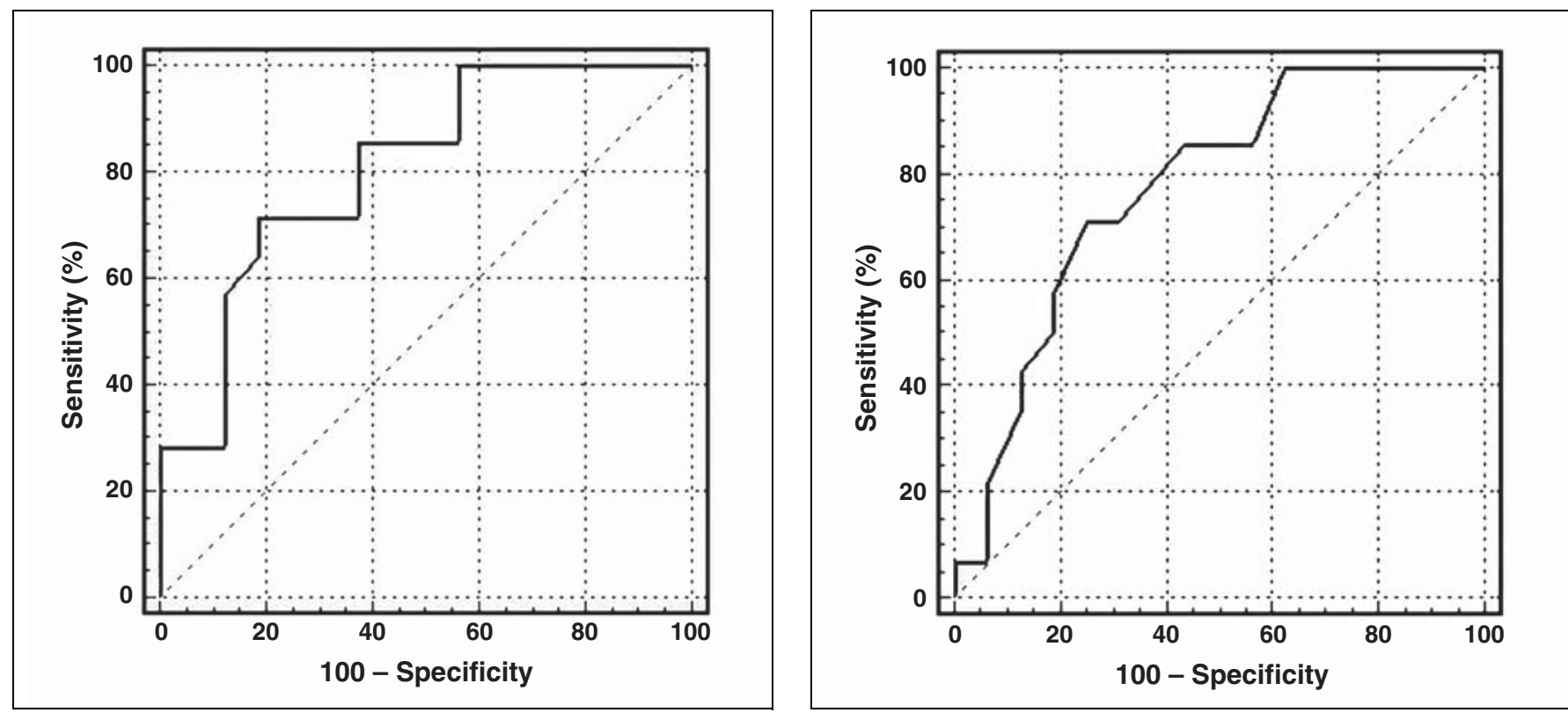

Fig. 2-Receiver operating characteristic (ROC) curves for relative ductal enhancement.

$\mathbf{A}$ and $\mathbf{B}, \mathrm{ROC}$ curves show relative ductal enhancement in portal venous phase $(\mathbf{A})$ and ductal wall thickness (B). Areas under ROC curves are 0.806 and 0.775, respectively. 


\section{CT Diagnosis of Cholangiocarcinoma Versus Periductal Fibrosis}

TABLE 2: CT Findings of Cholangiocarcinoma or Fibrosis in Patients with Hepatolithiasis

\begin{tabular}{|c|c|c|c|}
\hline \multirow[b]{2}{*}{ CT Finding } & \multicolumn{2}{|c|}{ No. $(\%)$ of Patients } & \multirow[b]{2}{*}{$p^{\mathrm{a}}$} \\
\hline & Cholangiocarcinoma $(n=14)$ & Fibrosis $(n=16)$ & \\
\hline Periductal soft-tissue density & $10(71.4)$ & $2(12.5)$ & 0.002 \\
\hline Ductal wall thickening (> $2.8 \mathrm{~mm})$ & $10(71.4)$ & $4(25)$ & 0.026 \\
\hline Portal vein obliteration & $6(42.9)$ & $1(6.3)$ & 0.031 \\
\hline Marked ductal dilatation & $4(28.6)$ & $3(18.8)$ & 0.675 \\
\hline Intrahepatic duct or common bile duct stone & $11(78.6)$ & $16(100)$ & 0.090 \\
\hline Lymph node enlargement & $6(42.9)$ & $1(6.3)$ & 0.031 \\
\hline Ascites & $2(14.3)$ & $1(6.3)$ & 0.586 \\
\hline High ductal enhancement in PVP (> $10 \mathrm{H}$ ) & $10(71.4)$ & $3(18.8)$ & 0.008 \\
\hline
\end{tabular}

Note-PVP = portal venous phase.

aFisher's exact test.

In this study, concomitant cholangiocarcinoma in patients with hepatolithiasis showed progressive enhancement from the HAP to PVP and appeared more conspicuous in the PVP than the HAP. This finding agrees with a previous study regarding cholangiocarcinoma in which periductal infiltrating intrahepatic cholangiocarcinoma appeared as a periductal soft-tissue density or a focal ductal wall thickening on enhanced CT, usually showing early or late enhancement or both $[13,16]$. There were two cases of benign biliary stricture $(12.5 \%, 2 / 16)$, which appeared as periductal low-attenuated soft-tissue-density lesions similar to malignant strictures in their enhancement pattern and were pathologically proven to be periductal fibrosis. Given that differentiating a periductal low-attenuated lesion of cholangiocarcinoma from that of benign fibrosis was not easy and that the incidence of periductal lesions of benign fibrosis is considerably lower than that of malignant stricture $(71.4 \%$, $10 / 14)$, the differential diagnosis of cholangiocarcinoma should be included when there is a periductal soft-tissue-density lesion on helical $\mathrm{CT}$ in patients with hepatolithiasis.
In an analysis of the ductal wall thickness, the cholangiocarcinoma group had a significantly thicker ductal wall than the fibrosis group ( $p=0.026)$ and cholangiocarcinoma also showed higher enhancement than normal duct wall on the PVP ( $p=0.008)$. There are a few reports regarding the usefulness of delayed phase imaging in the evaluation of cholangiocarcinoma and fibrosis $[16,26,27]$. Some authors have suggested that delayed postequilibrium phase tumor contrast enhancement is a typical feature of intrahepatic cholangiocarcinoma $[16,26]$ that may be attributed to the inherent dense fibrous stroma of the tumor. In addition, Keogan et al. [27] reported that delayed CT images were helpful for tumor characterization and improved tumor detection in the evaluation of hilar or intrahepatic cholangiocarcinoma and that the optimal time for acquisition of delayed images is 10-20 minutes after contrast medium injection. However, according to a study by Min et al. [7], a typical enhancement pattern of intrahepatic cholangiocarcinoma associated with hepatolithiasis on CT was progressive ductal wall enhancement in the arterial through the portal phases with decreased enhancement in the delayed phase.

Because in this study we did not acquire delayed CT images 10-20 minutes after contrast
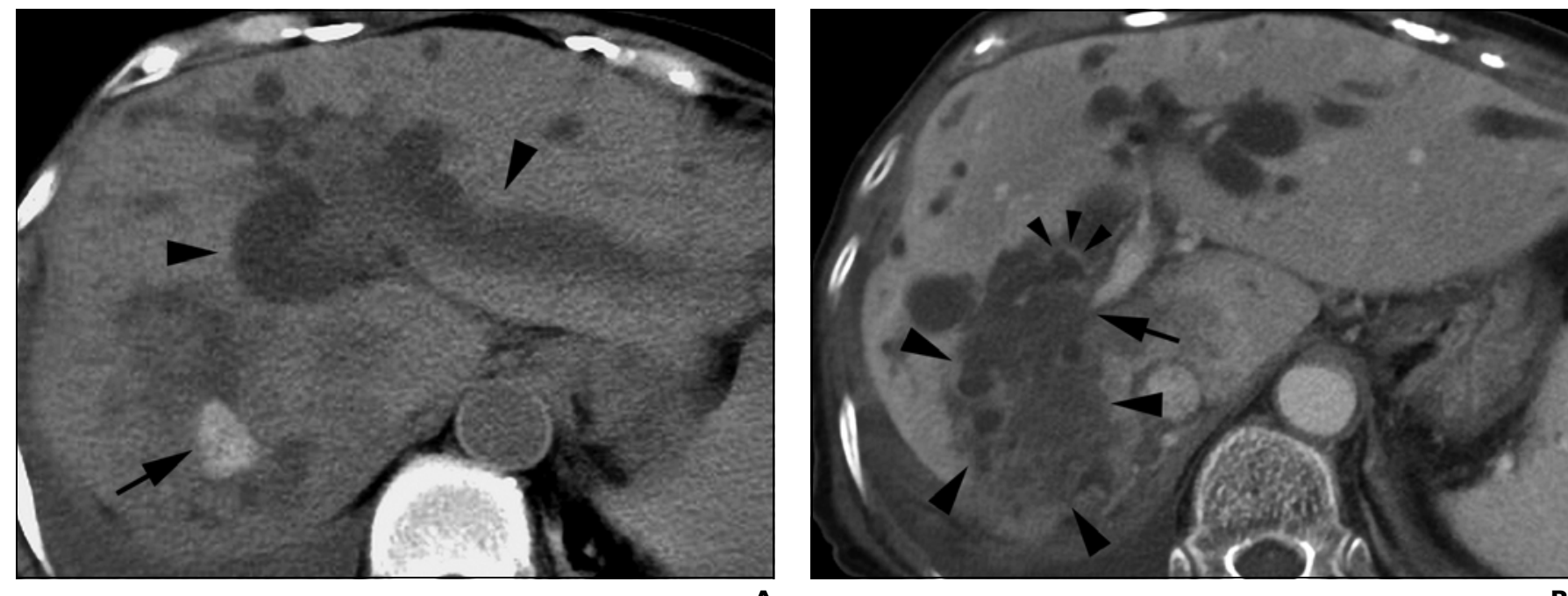

A

Fig. 3-75-year-old woman with hepatolithiasis and cholangiocarcinoma, which were confirmed by biopsy.

A, On unenhanced CT scan, there is an intrahepatic duct stone in the right lobe posterior segment of the liver (arrow). Both intrahepatic ducts (arrowheads) are dilated. Also note right pleural effusion.

B, Portal venous phase image shows periductal low-attenuated mass (larger arrowheads). Right intrahepatic duct (smaller arrowheads) is enhanced and the right portal vein (arrow) is obliterated. 


\section{Park et al.}

medium injection, we do not know the exact value of the delayed phase images for the differentiation of benign strictures from concomitant cholangiocarcinoma with hepatolithiasis. Considering the histologic feature of the abundant fibrotic component in both benign and malignant biliary strictures, cholangiocarcinoma containing a substantial fibrous component may be difficult to differentiate from fibrosis even on the delayed phase images [28, 29]. Further study will be necessary to determine the value of delayed phase imaging for differentiating benign from malignant biliary strictures.

Our study results indicate that the presence of portal vein obliteration was significant in differentiating cholangiocarcinoma from benign fibrotic stricture $(p=0.031)$. According to previous studies regarding hepatolithiasis $[30,31]$, the degree of portal vein narrowing correlated with the severity of liver atrophy and the pruned-tree appearance of portal veins reflected slight to moderate liver atrophy. Combined malignancy must be suspected, therefore, when there is complete portal vein obstruction associated with or without segmental lobar atrophy of the liver in hepatolithiasis.

Our study had several limitations. First, because this study was based on retrospective design, the precise correlation of the CT fea- tures with the histopathologic findings was not possible. Second, because our routine CT protocol for biliary diseases did not include delayed images - that is, longer than a 15minute delay - that could be helpful for the detection of cholangiocarcinoma [23], we were not able to assess the value of delayed enhancement of the lesions for differentiation between cholangiocarcinoma and fibrosis. Third, as to the analysis of lesion enhancement, we did not take account of the severity of the cholangitis when the CT scans were obtained and this may well have influenced the results of this study. Fourth, although we obtained multiphasic CT images using 5-mm

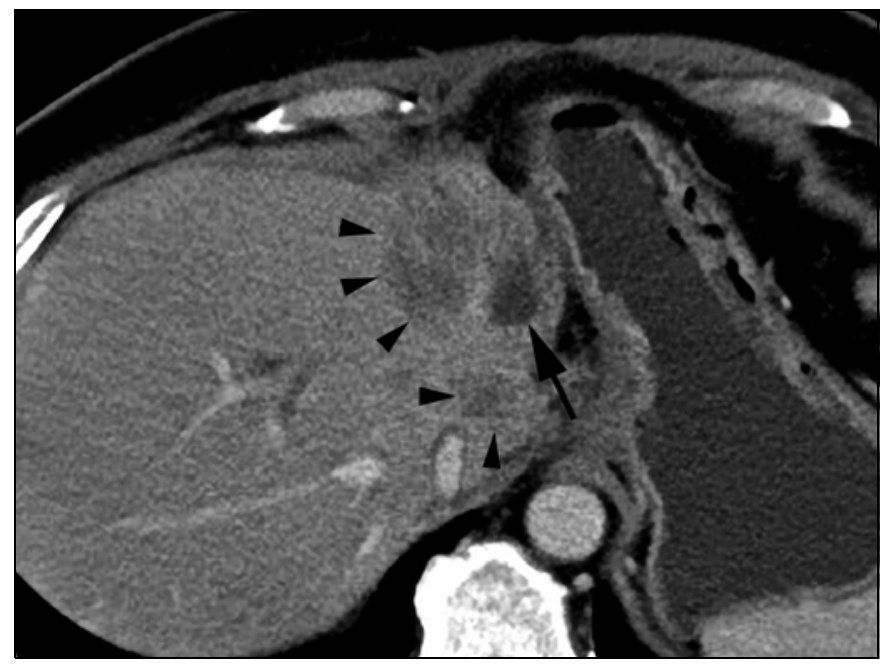

Fig. 4-53-year-old woman with hepatolithiasis and cholangiocarcinoma, which were confirmed by biopsy. She had undergone left lobectomy due to intrahepatic duct stones.

A, Portal venous phase (PVP) scan shows focal intrahepatic duct dilatation (arrow) and periductal low-attenuated soft-tissue lesions along dilated duct (arrowheads). B, On scan obtained more caudad to $\mathbf{A}$ on hepatic arterial phase, ductal wall (arrowheads) is thickened and enhanced well.

C, On PVP scan obtained at the same level as B, thickened ductal wall (arrowheads) is more strongly enhanced.
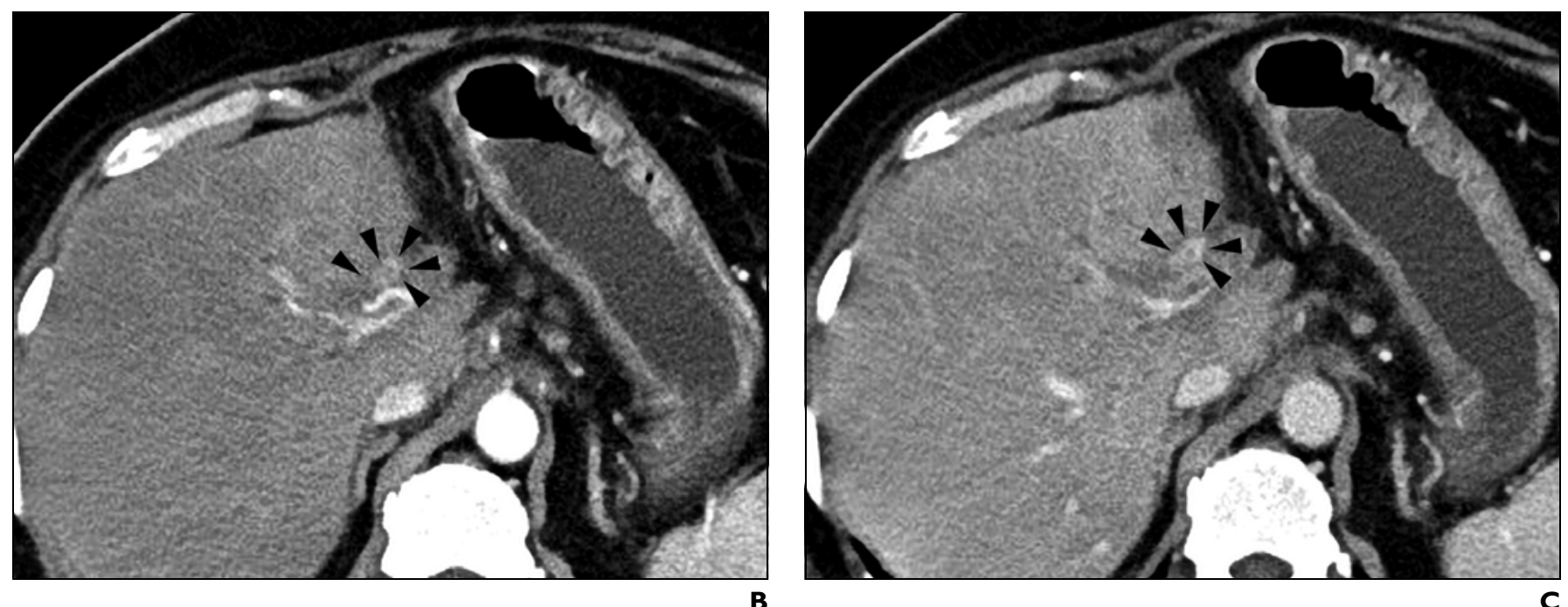


\section{CT Diagnosis of Cholangiocarcinoma Versus Periductal Fibrosis}
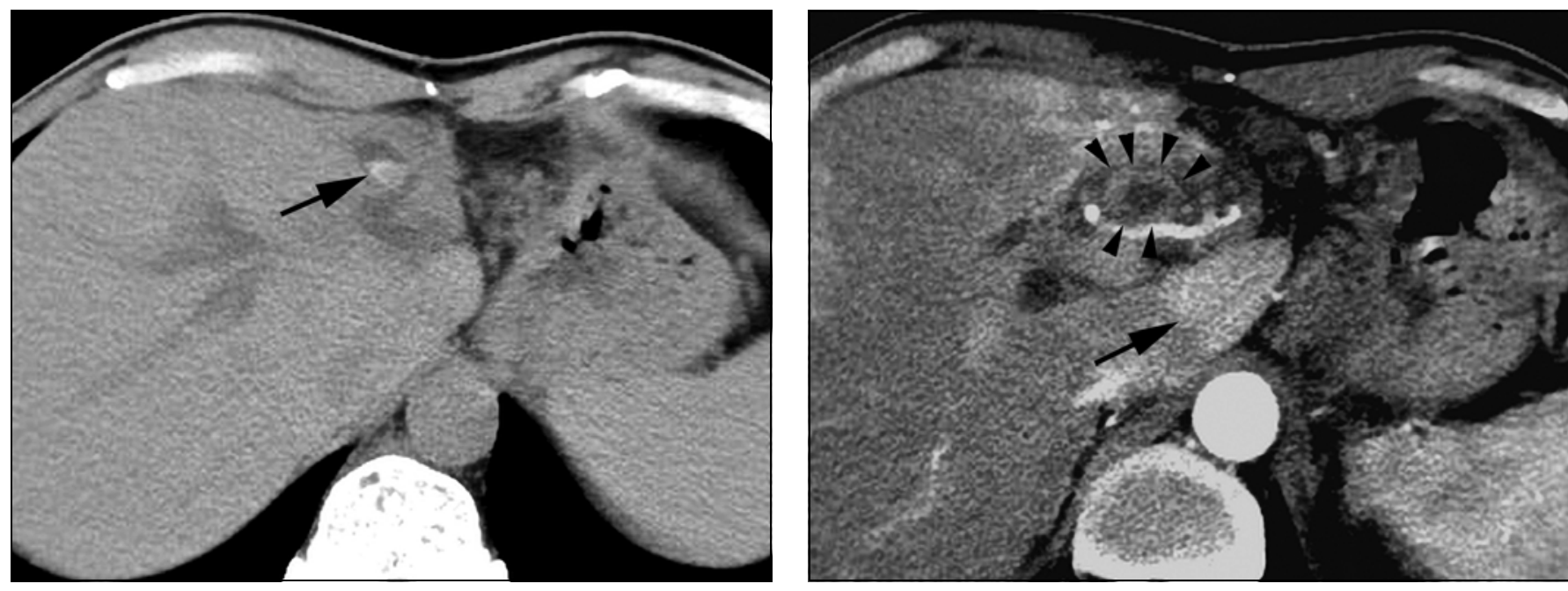

A
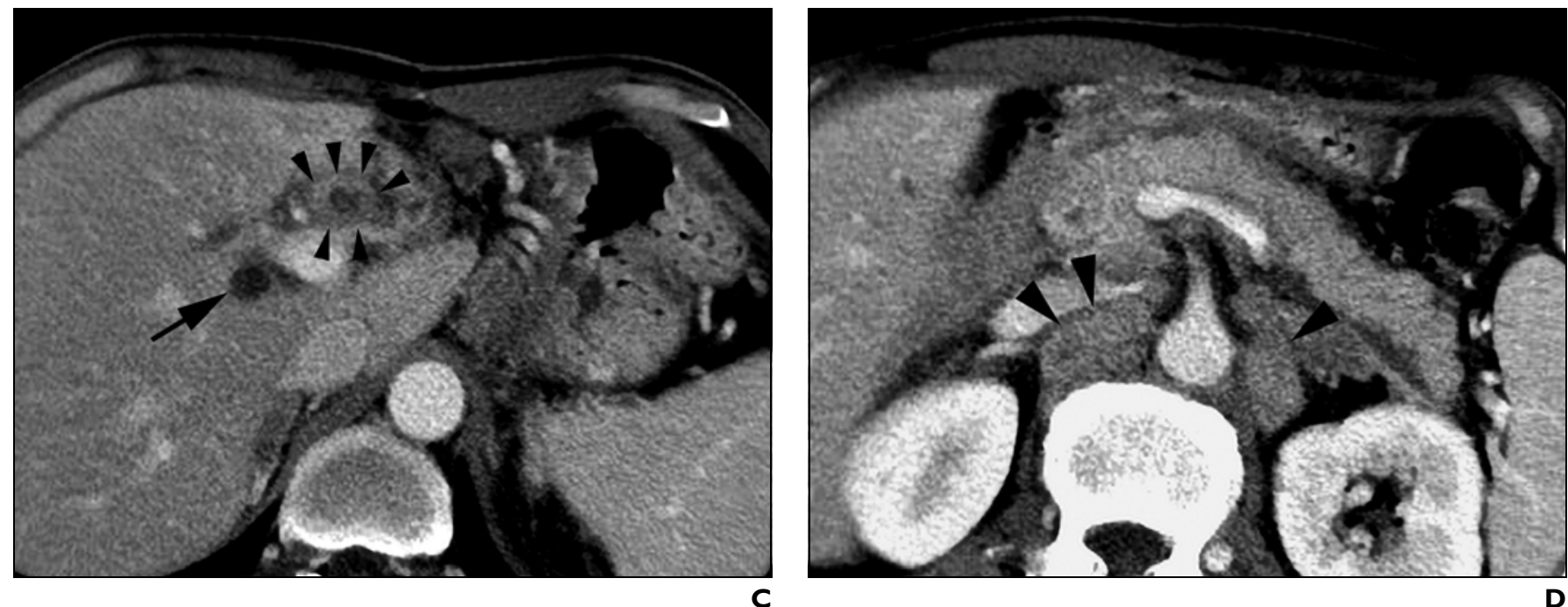

Fig. 5-59-year-old man with hepatolithiasis and cholangiocarcinoma, which were confirmed by common hepatic duct biopsy.

A, On unenhanced scan, left intrahepatic duct stone with dilated duct (arrow) is seen. Also note atrophied left lobe lateral segment of the liver.

B, Hepatic arterial phase scan shows dilated and thickened common hepatic duct with wall enhancement (arrowheads) and heterogeneous high-attenuated foci in the liver (arrow) suggesting transient hepatic attenuation difference.

C, On portal venous phase (PVP) scan, thickened wall of common hepatic duct (arrowheads) is more prominently enhanced than the wall of normal duct (arrow)

D, On PVP scan, aortocaval and paraaortic lymph nodes (arrowheads) are enlarged more than $1 \mathrm{~cm}$, suggesting lymph node metastasis.

collimation and 5-mm reconstructions, the scanners that were used are out of date; thus, the CT technique was not state-of-the-art. Last, only 3 of the 14 patients with cholangiocarcinoma underwent hepatic surgery; the others did not undergo surgery because of the advanced disease stage, expected inadequate hepatic reserve after resection, or medical co- morbidities. This indicates that the study population included many advanced cases, and the results may therefore be optimistically biased. However, this indirectly indicates that preoperative detection of cholangiocarcinoma in underlying hepatolithiasis is not easy and explains why our study is necessary. Despite the aforementioned problem of selection bias, we believe that our study results may help radiologists to differentiate the two diseases in patients with hepatolithiasis.

In summary, certain CT findings are helpful in detecting cholangiocarcinoma in patients with hepatolithiasis, and the combination of these CT findings may further improve accuracy in making the diagnosis. 


\section{Park et al.}

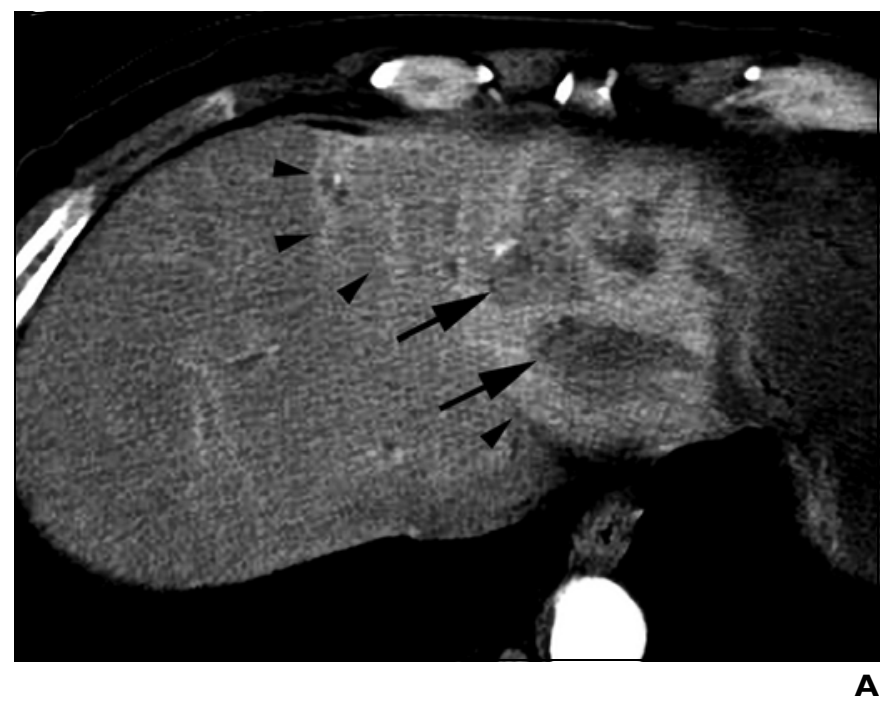

Fig. 6-76-year-old man with hepatolithiasis and periductal fibrosis, which were confirmed by the left lobectomy.

A, Hepatic arterial phase scan shows dilated left intrahepatic duct filled with lowattenuated material (arrows) that was identified as intraductal stones. Around the dilated duct is geographic high attenuation (arrowheads), suggesting transient hepatic attenuation difference, in atrophied left hepatic lobe.

B and C, On portal venous phase scans, there is dilated duct with intrahepatic duct stone (arrows, B) but no significant ductal wall thickening or enhancement (arrow, C) and intact portal vein (arrowheads, C).
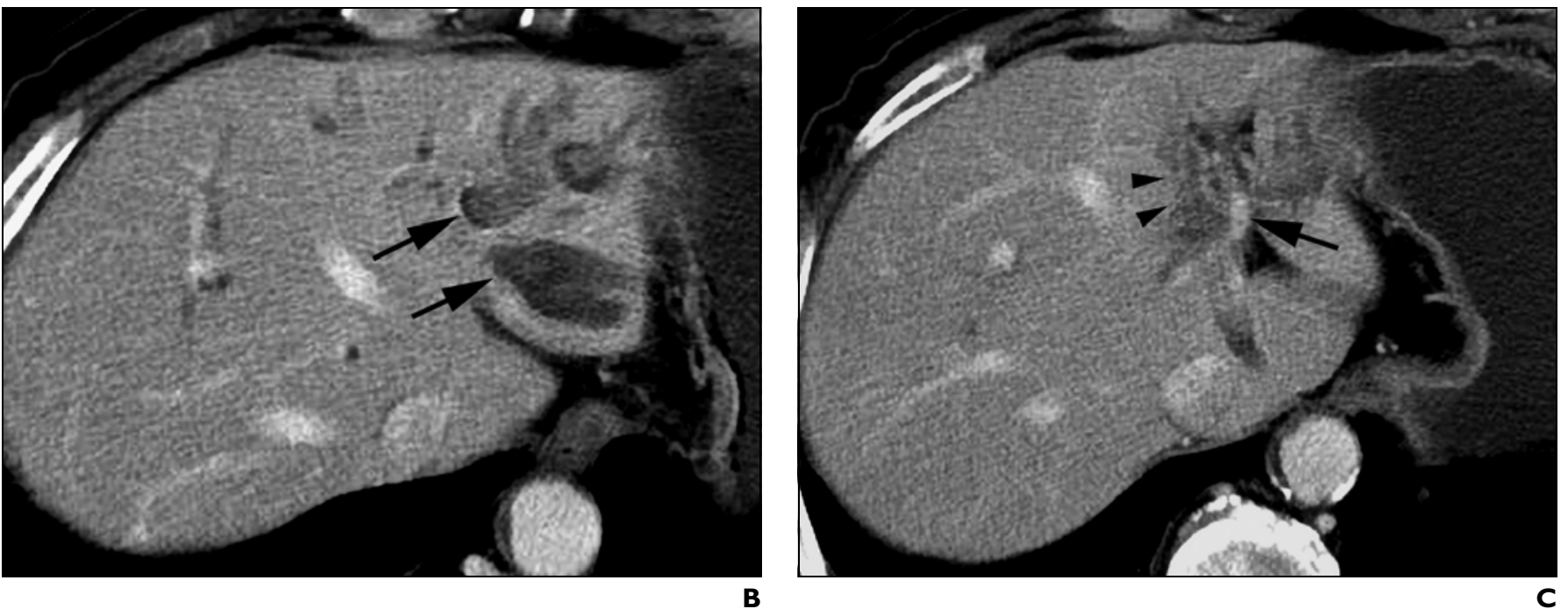

TABLE 3: Sensitivity and Specificity Values for CT Findings in the Diagnosis of Cholangiocarcinoma in Patients with Hepatolithiasis

\begin{tabular}{l|c|c|c|c}
\hline \multicolumn{1}{c|}{ CT Finding } & Sensitivity (\%) & $95 \% \mathrm{Cl}$ & Specificity (\%) & $95 \% \mathrm{Cl}$ \\
\hline Periductal soft-tissue density & $71.4(10 / 14)$ & $0.42-0.92$ & $87.5(14 / 16)$ & $0.62-0.98$ \\
Ductal wall thickening & $71.4(10 / 14)$ & $0.42-0.92$ & $75.0(12 / 16)$ & $0.48-0.93$ \\
Portal vein obliteration & $42.9(6 / 14)$ & $0.18-0.71$ & $93.8(15 / 16)$ & $0.70-1.00$ \\
Lymph node enlargement & $42.9(6 / 14)$ & $0.18-0.71$ & $93.8(15 / 16)$ & $0.70-1.00$ \\
High enhancement in PVP & $71.4(10 / 14)$ & $0.42-0.92$ & $18.8(3 / 16)$ & $0.50-0.94$ \\
\hline
\end{tabular}

TABLE 4: Combined CT Findings of

Cholangiocarcinoma in

Patients with

Hepatolithiasis

\begin{tabular}{c|c|c}
\hline $\begin{array}{c}\text { No. of CT } \\
\text { Findings }\end{array}$ & $\begin{array}{c}\text { Sensitivity } \\
(\%)\end{array}$ & $\begin{array}{c}\text { Specificity } \\
(\%)\end{array}$ \\
\hline 1 & $100(14 / 14)$ & $50.0(8 / 16)$ \\
2 & $100(14 / 14)$ & $87.5(14 / 16)$ \\
3 & $64.3(9 / 14)$ & $87.5(14 / 16)$ \\
4 & $21.4(3 / 14)$ & $100(16 / 16)$ \\
5 & $14.3(2 / 14)$ & $100(16 / 16)$ \\
\hline \multicolumn{3}{|c}{ Note-Data are the numbers of patients with at least } \\
one or more of any of the following lesion findings \\
on CT: periductal soft-tissue density; portal vein \\
obliteration; lymph node enlargement; and high \\
ductal enhancement on portal venous phase. \\
Numbers in parentheses are numbers of patients.
\end{tabular}




\section{CT Diagnosis of Cholangiocarcinoma Versus Periductal Fibrosis}

\section{Acknowledgment}

We thank Bonnie Hami for her editorial assistance in the preparation of this manuscript.

\section{References}

1. Dachman AH. Inflammatory cholangitis, parasitic diseases, primary biliary cirrhosis, and papillary (ampullary) stenosis. II. The gallbladder and biliary tract. In: Friedman AC, Dachman AH, eds. Radiology of the liver, biliary tract, and pancreas, 1 st ed. St. Louis, MO: Mosby, 1994:633-664

2. Lim JH. Oriental cholangiohepatitis: pathologic, clinical, and radiologic features. AJR 1991; 157:1-8

3. Chan FL, Man SW, Leong L, Fan ST. Evaluation of recurrent pyogenic cholangitis with CT: analysis of 50 patients. Radiology 1989; 170:165-169

4. Nakanuma Y, Terada T, Tanaka Y, Ohta G. Are hepatolithiasis and cholangiocarcinoma etiologically related? A morphological study of 12 cases of hepatolithiasis associated with cholangiocarcinoma. Virchows Arch A Pathol Anat Histopathol 1985; 406:45-58

5. Stain SC, Incabone R, Duthrie CR, et al. Surgical treatment of recurrent pyogenic cholangitis. Arch Surg 1995; 130:527-533

6. Koga A, Ichimiya H, Yamaguchi K, et al. Hepatolithiasis associated with cholangiocarcinoma: possible etiologic significance. Cancer 1985; 55:2826-2829

7. Min BC, Yoon KH, Kim CG, Roh BS, Won JJ. CT findings of intrahepatic cholangiocarcinoma associated with hepatolithiasis [in Korean]. J Korean Radiol Soc 1999; 41:525-531

8. Chen MF, Jan YY, Wang CS, et al. A reappraisal of cholangiocarcinoma in a patient with hepatolithiasis. Cancer 1993; 71:2461-2466

9. Su CH, Shyr YM, Lui WY, Peng FK. Hepatolithiasis associated with cholangiocarcinoma. Br J Surg

\section{7; 84:969-973}

10. Yamanaka N, Okamoto E, Ando T, et al. Clinicopathologic spectrum of resected extraductal massforming intrahepatic cholangiocarcinoma. Cancer 1995; 76:2449-2456

11. Jan YY, Chen MF. Surgical treatment of peripheral cholangiocarcinoma. Asian J Surg 1996; 19:105-111

12. Lee CC, Wu CY, Chen GH. Cholangiocarcinoma and hepatolithiasis: what is the impact of coexistence of hepatolithiasis on cholangiocarcinoma? J Gastroenterol Hepatol 2002; 17:1015-1020

13. Choi BI, Han JK, Shin YM, Baek SY, Han MC. Peripheral cholangiocarcinoma: comparison of MRI with CT. Abdom Imaging 1995; 20:357-360

14. Han JK, Choi BI, Kim AH, et al. Cholangiocarcinoma: pictorial essay of CT and cholangiographic findings. RadioGraphics 2002; 22:173-187

15. Kim TK, Choi BI, Han JK, Jang HJ, Cho SK, Han MC. Peripheral cholangiocarcinoma of the liver: two-phase spiral CT findings. Radiology 1997; 204:539-543

16. Lacomis JM, Baron RL, Oliver JH, Nalesnik MA, Federie MP. Cholangiocarcinoma: delayed CT contrast enhancement patterns. Radiology 1997; 203:98-104

17. Lee WJ, Lim HK, Jang KM, et al. Radiologic spectrum of cholangiocarcinoma: emphasis on unusual manifestations and differential diagnoses. RadioGraphics 2001; 21:S97-S116

18. Lim JH, Ko YT, Lee DH, et al. Cholangiocarcinoma associated with recurrent pyogenic cholangitis: sonographic and CT findings [in Korean]. J Korean Radiol Soc 1992; 28:115-119

19. Yoon KH, Ha HK, Lee JS, et al. Inflammatory pseudotumor of the liver in patients with recurrent pyogenic cholangitis: CT-histopathologic correlation. Radiology 1999; 211:373-379
20. Kubo S, Kinoshita H, Hirohashi K, Hamba H. Hepatolithiasis associated with cholangiocarcinoma. World J Surg 1995; 19:637-641

21. Lee JW, Han JK, Kim TK, et al. CT features of intraductal intrahepatic cholangiocarcinoma. AJR 2000; 175:721-725

22. Metz CE. Basic principles of ROC analysis. Semin Nucl Med 1978; 8:283-298

23. Chen MF, Jan YY, Hwang TL, Jeng LB, Yeh TS. Impact of concomitant hepatolithiasis on patients with peripheral cholangiocarcinoma. Dig Dis Sci 2000; 45:312-316

24. Nishihara K, Koga A, Sumiyoshi K, Kayashima K, Koso E. Intrahepatic calculi associated with cholangiocarcinoma. Jap J Surg 1986; 16:367-370

25. Anderson CD, Pinson CW, Berlin J, Chari RS. Diagnosis and treatment of cholangiocarcinoma. Oncologist 2004; 9:43-57

26. Itai Y, Ohtomo K, Kokubo T, et al. CT of hepatic masses: significance of prolonged and delayed enhancement. AJR 1986; 146:729-733

27. Keogan MT, Seabourn JT, Paulson EK, McDermott VG, Delong DM, Nelson RC. Contrast-enhanced CT of intrahepatic and hilar cholangiocarcinoma: delay time for optimal imaging. AJR 1997; 169:1493-1499

28. Lim JH. Cholangiocarcinoma: morphologic classification according to growth pattern and imaging findings. AJR 2003; 181:819-827

29. Yamashita Y, Takahashi M, Kanazawa S, et al. Hilar cholangiocarcinoma: an evaluation of subtypes with CT and angiography. Acta Radiol 1992; 33:351-355

30. Chou ST, Chan CW. Recurrent pyogenic cholangitis: a necropsy study. Pathology 1980; 12:415-428

31. Kusano S, Okada Y, Endo T, Yokoyama H, Ohmiya $\mathrm{H}$, Atari H. Oriental cholangiohepatitis: correlation between portal vein occlusion and hepatic atrophy. AJR 1992; 158:1011-1014 